University of Wollongong

Research Online

Australian Institute for Innovative Materials -

Papers

Australian Institute for Innovative Materials

$1-1-2015$

Observation of topological transition of Fermi surface from a spindle torus to a torus in bulk Rashba spin-split BiTeCl

Feixiang Xiang

University of Wollongong, fx963@uowmail.edu.au

Xiaolin Wang

University of Wollongong, xiaolin@uow.edu.au

Menno Veldhorst

University of Twente, University of New South Wales

S X. Dou

University of Wollongong, shi@uow.edu.au

Michael S. Fuhrer

Monash University

Follow this and additional works at: https://ro.uow.edu.au/aiimpapers

Part of the Engineering Commons, and the Physical Sciences and Mathematics Commons

Research Online is the open access institutional repository for the University of Wollongong. For further information contact the UOW Library: research-pubs@uow.edu.au 


\title{
Observation of topological transition of Fermi surface from a spindle torus to a torus in bulk Rashba spin-split BiTeCl
}

\author{
Abstract \\ The recently observed large Rashba-type spin splitting in the $\mathrm{BiTeX}(\mathrm{X}=\mathrm{I}, \mathrm{Br}, \mathrm{Cl})$ bulk states enables \\ observation of the transition in Fermi surface topology from spindle torus to torus with varying the carrier \\ density and offers an ideal platform for achieving practical spintronic applications and realizing nontrivial \\ phenomena such as topological superconductivity and Majorana fermions. Here we use Shubnikov-de \\ Haas oscillations to investigate the electronic structure of the bulk conduction band of $\mathrm{BiTeCl}$ single \\ crystals with different carrier densities. We observe the topological transition of the Fermi surface (FS) \\ from a spindle torus to a torus. The Landau-level fan diagram reveals the expected nontrivial $\pi$ Berry \\ phase for both the inner and outer FSs. Angle-dependent oscillation measurements reveal three- \\ dimensional FS topology when the Fermi level lies in the vicinity of the Dirac point. All the observations \\ are consistent with large Rashba spin-orbit splitting in the bulk conduction band.
}

\section{Keywords}

split, spin, rashba, bulk, bitecl, torus, spindle, surface, fermi, transition, topological, observation

\section{Disciplines}

Engineering | Physical Sciences and Mathematics

\section{Publication Details}

Xiang, F., Wang, X., Veldhorst, M., Dou, S. \& Fuhrer, M. S. (2015). Observation of topological transition of Fermi surface from a spindle torus to a torus in bulk Rashba spin-split BiTeCl. Physical Review B:

Condensed Matter and Materials Physics, 92 (3), 035123-1-035123-12. 


\title{
Observation of topological transition of Fermi surface from a spindle torus to a torus in bulk Rashba spin-split BiTeCl
}

\author{
Fei-Xiang Xiang, ${ }^{1}$ Xiao-Lin Wang, ${ }^{1,}{ }^{*}$ Menno Veldhorst, ${ }^{2}$ Shi-Xue Dou, ${ }^{1}$ and Michael S. Fuhrer ${ }^{3}$ \\ ${ }^{1}$ Institute for Superconducting and Electronic Materials, Australian Institute for Innovative Materials, University of Wollongong, \\ Innovation Campus, North Wollongong, New South Wales 2500, Australia \\ ${ }^{2}$ Centre for Quantum Computation and Communication Technology, School of Electrical Engineering and Telecommunications, \\ The University of New South Wales, Sydney, New South Wales 2052, Australia \\ ${ }^{3}$ School of Physics, Monash University, Clayton, Victoria 3800, Australia
}

(Received 17 March 2015; published 13 July 2015)

\begin{abstract}
The recently observed large Rashba-type spin splitting in the $\operatorname{BiTe} X(X=\mathrm{I}, \mathrm{Br}, \mathrm{Cl})$ bulk states enables observation of the transition in Fermi surface topology from spindle torus to torus with varying the carrier density and offers an ideal platform for achieving practical spintronic applications and realizing nontrivial phenomena such as topological superconductivity and Majorana fermions. Here we use Shubnikov-de Haas oscillations to investigate the electronic structure of the bulk conduction band of BiTeCl single crystals with different carrier densities. We observe the topological transition of the Fermi surface (FS) from a spindle torus to a torus. The Landau-level fan diagram reveals the expected nontrivial $\pi$ Berry phase for both the inner and outer FSs. Angle-dependent oscillation measurements reveal three-dimensional FS topology when the Fermi level lies in the vicinity of the Dirac point. All the observations are consistent with large Rashba spin-orbit splitting in the bulk conduction band.
\end{abstract}

DOI: 10.1103/PhysRevB.92.035123

PACS number(s): 75.47.-m, 71.18.+y

\section{INTRODUCTION}

Spin-orbit coupling (SOC) is a relativistic effect present in a system with broken symmetry, where a charged particle moving in an electric field experiences an effective magnetic field which interacts with its spin [1,2]. In solids, electrons move in a crystal potential, and if there is a potential gradient, effective SOC arises [2] and manifests itself in the spin-split band structure. Such spin splitting was first described by the Dresselhaus [3] and Rashba [4] model in the zinc-blende and the wurtzite structure, respectively, and later by the BychkovRashba model [5] at surfaces and interfaces. Although large spin splitting has been observed at the surface of heavy metals, it remains small in conventional semiconductors.

Recently, large Rashba-type spin splitting has been observed in the bulk bands of $\operatorname{BiTe} X(X=\mathrm{I}, \mathrm{Br}, \mathrm{Cl})$ polar semiconductors due to the broken inversion symmetry and charge polarity in the bulk [6-11]. The high energy scale of the Rashba effect in $\mathrm{BiTe} X$ provides opportunities for achieving practical spintronic applications $[12,13]$ and realizing nontrivial phenomena such as the intrinsic spin Hall effect [14], noncentrosymmetric exotic superconductivity $[15,16]$, Majorana fermions $[17,18]$, and topological transitions of the Fermi surface (FS) $[9,19,20]$. Among them, the topological transition of the FS takes place when the Fermi level is tuned down through the band-crossing point (Dirac point), as shown in Fig. 1(a). When the Fermi energy $E_{F}$ is larger than the Rashba energy $E_{R}$, i.e., $E_{F}>E_{R}$, the FS is a spindle torus [(iv) of Fig. 1(a)]. When $E_{F}=E_{R}$, the spindle inner FS (IFS) disappears, and only the outer FS (OFS) remains $[(\mathrm{v})$ of Fig. 1(a)]. When $E_{F}<E_{R}$, the FS becomes a ring torus [(vi) of Fig. 1(a)] [9]. In this transition, both the number and type of the FSs change by 1 [9]. Furthermore, due to the opposite

\footnotetext{
*xiaolin@uow.edu.au
}

spin helicity of the OFS and IFS, the ratio of carrier densities with opposite spin helicity changes with this transition. When $E_{F}>E_{R}$, two types of carriers with opposite spin helicity are present [(i) of Fig. 1(a)]. When $E_{F} \leqslant E_{R}$, the spin helicity of all the carriers is the same [(ii) and (iii) of Fig. 1(a)]. Therefore such a transition would be an important step toward exploring spin-dependent transport and other exotic physical phenomena in the low-carrier-density regime $[9,19,20]$. In particular, it is highly desirable to tune the Fermi level into the vicinity of the Dirac point at zero momentum in the Rashba system, since schemes to realize Majorana fermions [18] involve opening a small energy gap at the Dirac point to realize a single spin nondegenerate band. Furthermore, the spin polarization of current is largest in the vicinity of the Dirac point [20,21] and hence, such materials could be used as spin injectors, as has been proposed with topological insulators [22,23], but the surface-dominated transport is not required in this case.

Magnetotransport is a powerful method to study the electronic properties of materials such as topological insulators $[24,25]$. In particular, the Shubnikov-de Haas $(\mathrm{SdH})$ oscillation can probe the electronic structure, reveal information on the FS topology [26,27], and access the Berry phase [25,28-31], so it is highly suitable for investigating topological transitions of the Fermi surface and detecting the potential topological surface states. Although $\mathrm{BiTeCl}$ has smaller spin splitting than BiTeI, it exhibits a larger band gap and more isotropic spin splitting [10], which are very desirable for transport measurements.

In this paper, using the $\mathrm{SdH}$ effect, we observe a transition from two sets of oscillations to one as the carrier density varies in $\mathrm{BiTeCl}$ single crystals. The Landau-level (LL) fan diagram reveals the nontrivial $\pi$ Berry phase both in the IFS and the OFS. We also resolve the three-dimensional (3D) FS topology when the Fermi level lies in the vicinity of the Dirac point by angle-dependent oscillation measurements. All the observations are consistent with a topological transition of the 
FS from a spindle torus to a torus in the large Rashba spin-split conduction band of $\mathrm{BiTeCl}$.

\section{EXPERIMENTAL DETAILS}

Single crystals of $\mathrm{BiTeCl}$ were grown by the self-flux method according to the $\mathrm{Bi}_{2} \mathrm{Te}_{3}-\mathrm{BiCl}_{3}$ binary phase diagram. $\mathrm{Bi}_{2} \mathrm{Te}_{3}$ was synthesized from high-purity $(5 \mathrm{~N}) \mathrm{Bi}$ and Te powders. $\mathrm{Bi}_{2} \mathrm{Te}_{3}$ and $\mathrm{BiCl}_{3}(5 \mathrm{~N})$ powders were weighed out with the molar ratio of 1:9 and thoroughly ground together, with these operations carried out in an oxygen- and moisturemonitored glovebox to prevent the deliquescence of $\mathrm{BiCl}_{3}$. The mixture of powders was then loaded into a quartz tube and sealed under vacuum before heating to above $420{ }^{\circ} \mathrm{C}-500{ }^{\circ} \mathrm{C}$ over several hours. The temperature was maintained for $12 \mathrm{~h}$, and then the samples were slowly cooled to $200^{\circ} \mathrm{C}$ over several days. The platelike crystals were obtained by chemically removing the residual flux of $\mathrm{BiCl}_{3}$.

Single-crystal samples of $\mathrm{BiTeCl}$ with shining surfaces were cleaved from the as-grown crystals and used for standard four-probe transport measurements. In some cases, a six-probe Hall measurement was employed to obtain the longitudinal resistance, $R_{x x}$, and the Hall resistance, $R_{x y}$, simultaneously. Gold wires were attached to the sample surface by silver epoxy, which was cured at room temperature before the measurements to ensure Ohmic contacts. The magnetic field $B$ was applied perpendicular to the sample surface and varied up to $13.5 \mathrm{~T}$, except for the angle-dependent measurements. The temperature for magnetotransport measurements was $2.5 \mathrm{~K}$, except for measurements of the $\mathrm{SdH}$ oscillations at various temperatures.

\section{RESULTS AND DISCUSSION}

\section{A. Observation of the topological transition of the Fermi surface}

In contrast to BiTeI, the Rashba spin-orbit splitting in $\mathrm{BiTeCl}$ occurs in the $\Gamma K-\Gamma M$ plane of momentum space, denoted as the $k_{\|}$plane, where momentum is along the $z$ direction, $k_{z}=0[10,11,32,33]$. The energy-momentum dispersion can be described by the following equation, assuming a parabolic band:

$$
E_{ \pm}(k)=\frac{\hbar^{2}}{2 m^{*}}\left(k \pm k_{0}\right)^{2}
$$

where $k=\sqrt{k_{x}^{2}+k_{y}^{2}}, k_{0}$ is the momentum offset caused by the Rashba spin splitting, $m^{*}$ is the effective mass of the electrons, and $\hbar$ is Planck's constant divided by $2 \pi$. Besides the $k_{0}$, the other two Rashba parameters are the Rashba energy, $E_{R}=\hbar^{2} k_{0}^{2} / 2 m^{*}$, and the Rashba constant, $\alpha_{R}=2 E_{R} / k_{0}$, which represent the energy when $k=0$ and the strength of the Rashba effect, respectively. The spin-split conduction band dispersion of $\mathrm{BiTeCl}$ near the $\Gamma$ point is shown on the left of Fig. 1(a), and the right of Fig. 1(a) shows the 3D FS when the Fermi energy $E_{F}$ has different values. When $E_{F}>E_{R}$, the $3 \mathrm{D}$ FS is a spindle torus, and both the IFS (spindle) and (a)

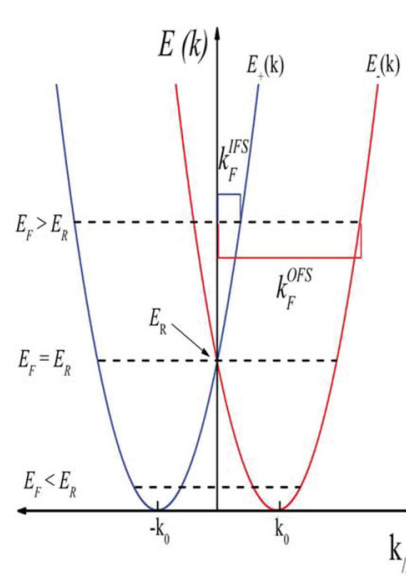

(b)

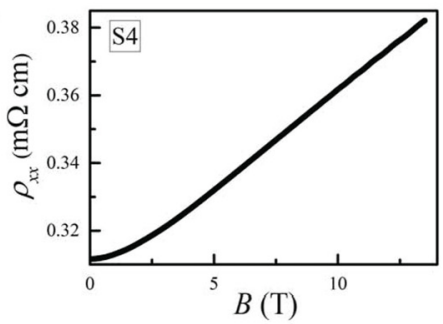

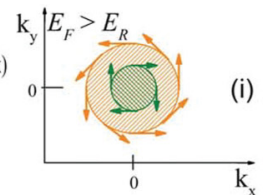

(i)

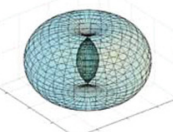

(iv)

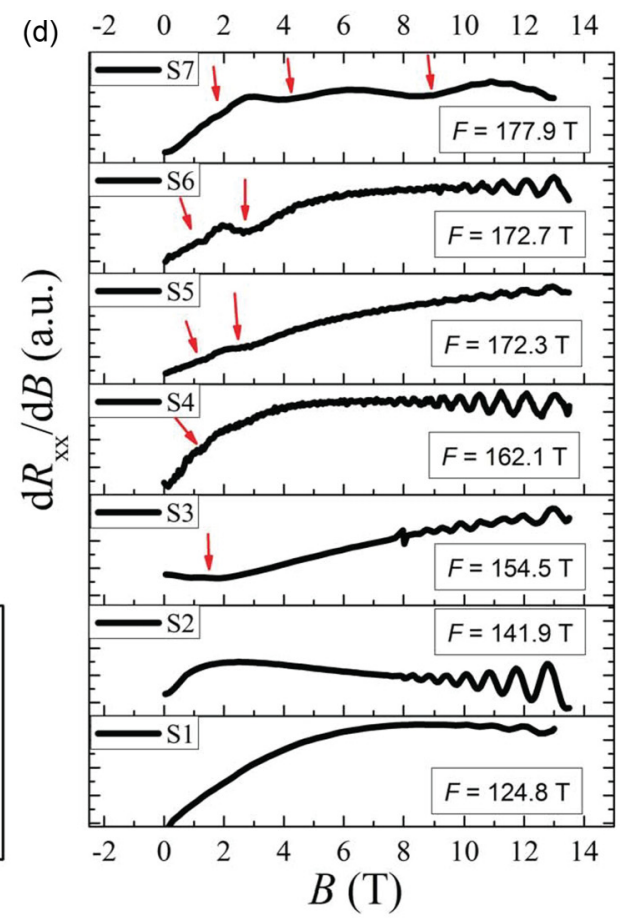

FIG. 1. (Color online) (a) (Left) Energy-momentum dispersion in the Rashba spin-split conduction band. The dashed lines represent different Fermi energies $E_{F}$. The blue and red colors indicate two opposite spin directions. (Middle) The constant energy contours at the $k_{\|}$ plane and (right) the three-dimensional FS for $E_{F}>E_{R}$ [(i) and (iv)], $E_{F}=E_{R}$ [(ii) and (v)], and $E_{F}<E_{R}$ [(iii) and (vi)], respectively. The orange and green colors in the middle of Fig. 1(a) indicate different spin helicities, and the arrows show the spin direction. Longitudinal resistivity $\rho_{x x}$ (b) and Hall resistivity $\rho_{x y}$ (c) as functions of magnetic field $B$ for sample S4. (d) Evolution of the topological transition as the Fermi level shifts from well above the Dirac point down to the vicinity of the Dirac point. The arrows indicate the oscillation periods from the IFS that can be observed. 
OFS (torus) are present [(iv) of Fig. 1(a)]. While in a small Rashba spin-split system, the IFS and OFS result in beating patterns in the $\mathrm{SdH}$ oscillations; in a giant Rashba spin-split system, the two sets of oscillations are thoroughly decoupled from each other [29]. When $E_{F} \leqslant E_{R}$, the IFS vanishes and the FS is simply a torus, i.e., only the OFS is present [(v) and (vi) of Fig. 1(a)]. Thus it is expected that the two sets of oscillations represent a transition to a single frequency in the Shubnikov-de Haas oscillation measurement when the Fermi level approaches the Dirac point, corresponding to the topological transition of FS from spindle torus to torus.

$\mathrm{BiTeCl}$ is a degenerate semiconductor due to the self-doping effect (nonstoichiometric effect or formation of defects), which is similar to what occurs in the topological insulators such as $\mathrm{Bi}_{2} \mathrm{Se}_{3}$ and $\mathrm{Bi}_{2} \mathrm{Te}_{3}$. Because the self-doping effect depends on the temperature gradient along the quartz tube during the single-crystal growth, the carrier density of the crystal can vary in different positions in the quartz tube. To observe the expected topological transition of the FS as the Fermi level approaches the Dirac point, a group of single-crystal samples with various carrier concentrations was selected from different positions in the tube. The samples are denoted as S1-S7 and are ordered according to their increasing OFS oscillation frequency. Figures 1(b) and 1(c) show the typical longitudinal resistivity $\left(\rho_{x x}\right)$ and Hall resistivity $\left(\rho_{x y}\right)$ of the

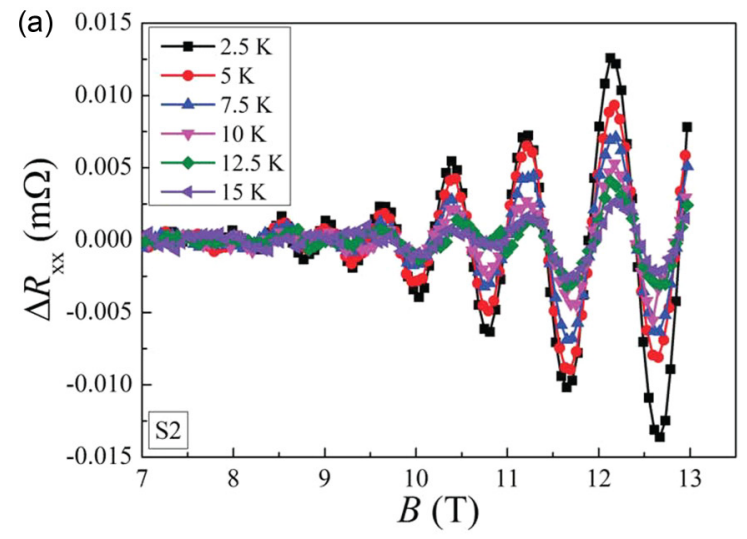

(c)

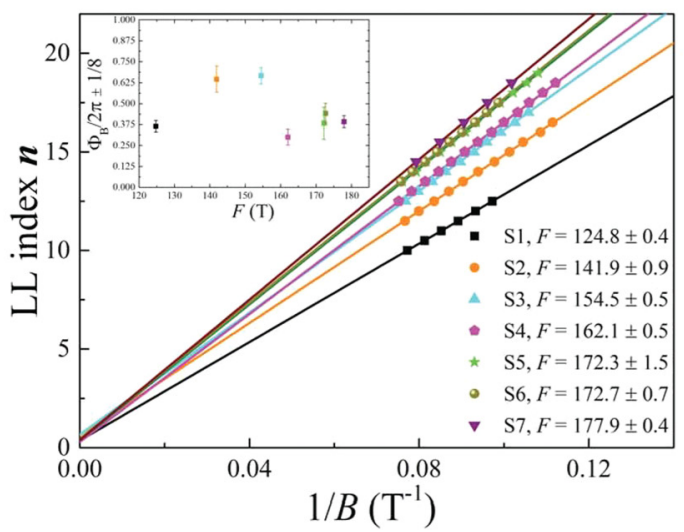

samples. The negative linear slope in Fig. 1(c) indicates that the dominant carriers are electrons. The calculated carrier density is $8.87 \times 10^{18} \mathrm{~cm}^{-3}$. A clear evolution of the transition from two sets of oscillations to a single-frequency oscillation is shown in Fig. 1(d). From S7 to S1 the oscillations from the IFS indicated by the red arrows gradually disappear, and only oscillations from the OFS are left, consistent with the topological transition of the FS described above $[9,19]$.

\section{B. Standard Shubnikov-de Haas oscillation analysis}

To deduce the electronic structure via standard $\mathrm{SdH}$ oscillation analysis, the Lifshitz-Kosevich (LK) formula is used as follows [26,27,29]:

$$
\begin{aligned}
\frac{\Delta \rho}{\rho_{0}}= & \frac{5}{2}\left(\frac{B}{2 F}\right)^{\frac{1}{2}} \frac{2 \pi^{2} k_{B} T m^{*} / \hbar e B}{\sinh \left(2 \pi^{2} k_{B} T m^{*} / \hbar e B\right)} \mathrm{e}^{-\frac{2 \pi^{2} k_{B} T_{D^{m}}}{\hbar e B}} \\
& \times \cos 2 \pi\left(\frac{F}{B}+\frac{1}{2}-\frac{\Phi_{B}}{2 \pi}+\delta\right),
\end{aligned}
$$

where $F$ is the oscillation frequency, $k_{B}$ the Boltzmann constant, $e$ the elementary charge, $T$ the temperature, $T_{D}$ the Dingle temperature, $\Phi_{\mathrm{B}}$ the Berry phase, and $\delta$ the phase shift determined by the dimensionality. Figure 2(a) shows the OFS $\mathrm{SdH}$ oscillations of $\mathrm{S} 2$ at various temperatures after subtracting
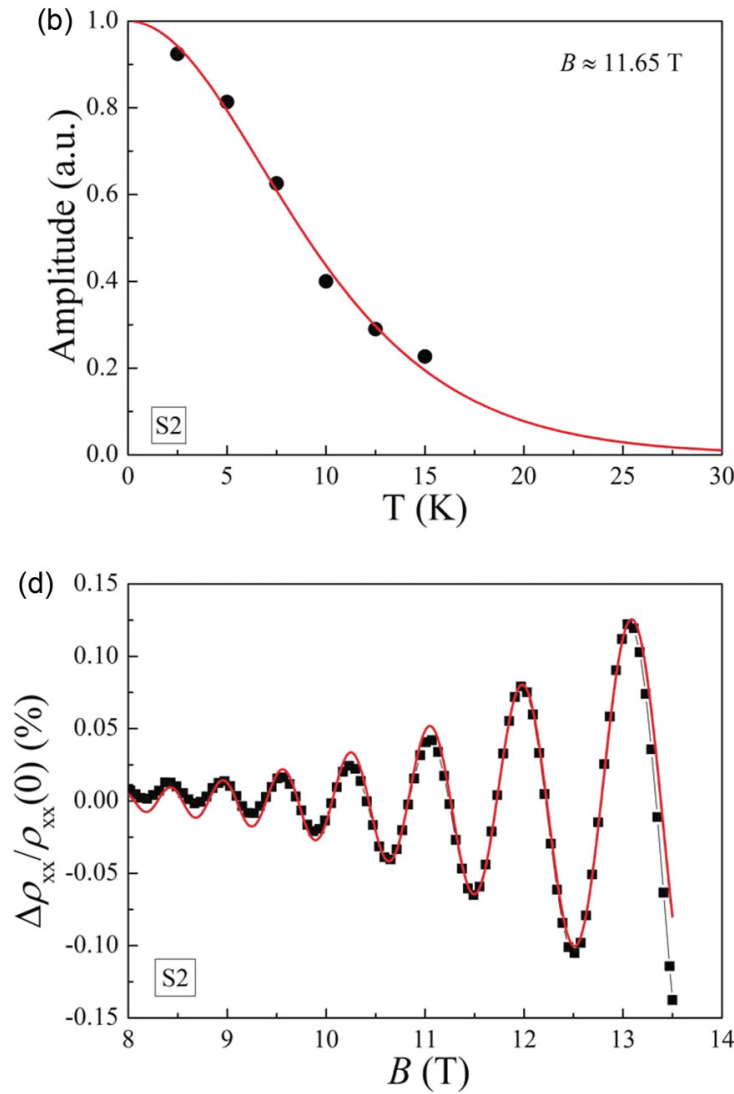

FIG. 2. (Color online) (a) OFS SdH oscillations of S2 at various temperatures after subtracting the background. (b) Temperature dependence of the OFS oscillation amplitude of S2. Fitting with the thermal damping factor yields the effective mass $m^{*}$. (c) LL fan diagram used to obtain the oscillation frequencies $F$ and the phase factors of the OFSs for samples S1-S7. The solid symbols denote LL indices for the minima and maxima of the $\mathrm{SdH}$ oscillations. The solid lines are the linear fits to the experimental data. The values of the intercepts of the fitting lines with the LL index axis are shown in the inset. The error bars in the inset indicate the standard deviation of the fitting errors. (d) OFS SdH oscillations of $\mathrm{S} 2$ fitted by the LK formula, which yields the Dingle temperature. 
TABLE I. Parameters determined from the SdH oscillations and the Rashba model. $m^{* O F S}$ is the effective mass of the OFS. $F^{\mathrm{OFS}}$ and $F^{\mathrm{IFS}}$ are oscillation frequencies from the OFS and IFS, which yield $k_{F}^{\mathrm{OFS}}$ and $k_{F}^{\mathrm{IFS}}$, via the Onsager relation. $T_{D}^{\mathrm{OFS}}$ is the Dingle temperature of the OFS. $E_{F}$ is calculated by the Rashba model. The carrier density $n$ is calculated with the $3 \mathrm{D}$ model, $\left(1 / 3 \pi^{2}\right)(2 e F / \hbar)^{3 / 2}$. $\mu$ is the carrier mobility calculated by $e \hbar / 2 m^{*} \pi k_{B} T_{D}$.

\begin{tabular}{|c|c|c|c|c|c|c|c|c|c|}
\hline Sample & $\begin{array}{c}m^{* \mathrm{OFS}} \\
\left(m_{e}\right)\end{array}$ & $\begin{array}{c}F^{\mathrm{OFS}} \\
(\mathrm{T})\end{array}$ & $\begin{array}{c}T_{D}^{\mathrm{OFS}} \\
(\mathrm{K})\end{array}$ & $\begin{array}{c}k_{F}^{\mathrm{OFS}} \\
\left(10^{-3} \AA\right)\end{array}$ & $\begin{array}{c}\mu^{\mathrm{OFS}} \\
\left(\mathrm{cm}^{2} \mathrm{~V}^{-1} \mathrm{~s}^{-1}\right)\end{array}$ & $\begin{array}{l}F^{\mathrm{IFS}} \\
(\mathrm{T})\end{array}$ & $\begin{array}{c}k_{F}^{\mathrm{IFS}} \\
\left(10^{-3} \AA\right)\end{array}$ & $\begin{array}{c}\mathrm{E}_{\mathrm{F}} \\
(\mathrm{meV})\end{array}$ & $\begin{array}{c}n \\
\left(10^{18} \mathrm{~cm}^{-3}\right)\end{array}$ \\
\hline S1 & 0.203 & 124.8 & 22.98 & 61.59 & 458.5 & & & 18.38 & 7.9 \\
\hline $\mathrm{S} 2$ & 0.191 & 141.9 & 17.5 & 65.69 & 640.2 & & & 23.61 & 9.58 \\
\hline S3 & 0.199 & 154.5 & 20 & 68.53 & 537.4 & & & 27.61 & 10.88 \\
\hline S4 & 0.194 & 162.1 & 20.5 & 70.20 & 537.8 & & & 30.11 & 11.69 \\
\hline S5 & 0.194 & 172.3 & 21.05 & 72.37 & 522.9 & 2.81 & 9.24 & 33.52 & 12.81 \\
\hline S6 & 0.197 & 172.7 & 15.53 & 72.45 & 699.1 & & & 33.65 & 12.86 \\
\hline S7 & & 177.9 & & 73.54 & & 5.93 & 13.43 & 35.44 & 13.45 \\
\hline
\end{tabular}

the background. Fitting the temperature dependence of the OFS oscillation amplitudes of S2 around $11.65 \mathrm{~T}$ with the thermal damping factor $\frac{2 \pi^{2} k_{B} T m^{*} / \hbar e B}{\sinh \left(2 \pi^{2} k_{B} T m^{*} / \hbar e B\right)}$ from Eq. (2), as shown in Fig. 2(b), yields the effective mass $m^{*}=0.191 \pm 0.005 m_{\mathrm{e}}$, where $m_{\mathrm{e}}$ is the free electron mass. Because $\rho_{x x}<\rho_{x y}$, as shown in Figs. 1(b) and 1(c), the minima and maxima of the $\mathrm{SdH}$ oscillations are assigned as integer $(n)$ and half-integer $(n+1 / 2)$ LL indices, respectively. Linear fits of the LL indices vs $1 / B$ of S1-S7 in the LL fan diagram of Fig. 2(c) yield the oscillation frequency $F$ (slopes of the fit lines) and the phase fac- tor $-\frac{\Phi_{B}}{2 \pi}+\delta$ (intercepts with the LL index axis) for each sample. Taking the value $\delta= \pm \frac{1}{8}$ for our 3D system [26,27,29], the range of the intercepts from 0.375 to 0.625 indicates a nontrivial $\pi$ Berry phase. The inset of Fig. 2(c) shows the Berry phases for S1-S7, and nearly all of them are located in the range from $0.375-0.625$. After obtaining $m^{*}, F$, and the phase factor, we fit the OFS SdH oscillation data of S2 with Eq. (2) to obtain the Dingle temperature $T_{D}$. As shown in Fig. 2(d), the LK formula fits well with the experimental data, resulting in $T_{D}=17.6 \pm 0.12 \mathrm{~K}$. The same analysis process was applied
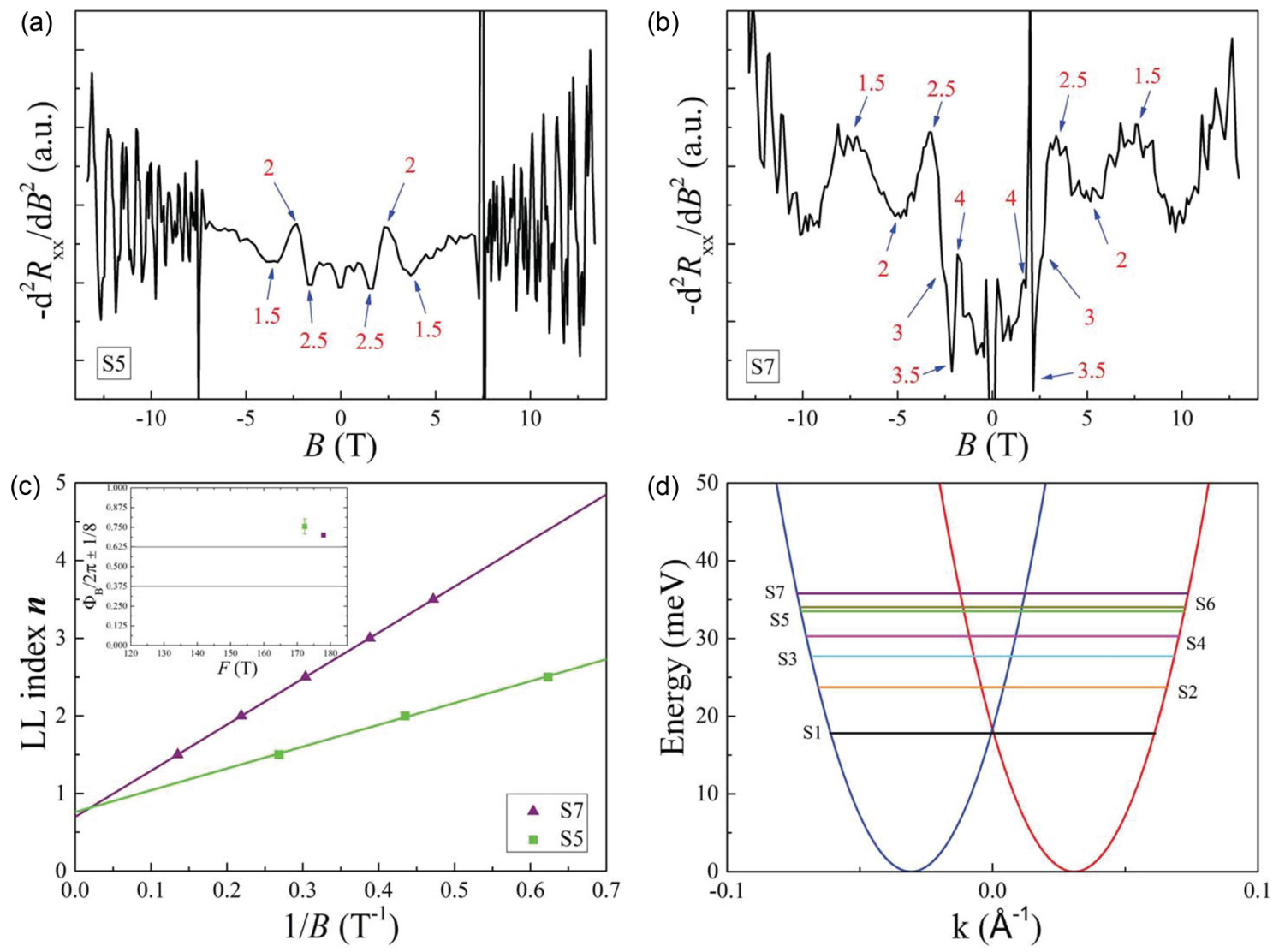

FIG. 3. (Color online) $-d^{2} R_{x x} / d B^{2}$ as a function of $B$ for (a) S5 and (b) S7. The blue arrows indicate the positions of the integer and half-integer LLs. (c) LL fan diagram for IFS of S5 and S7. The inset shows the values of the intercepts of the fitting lines with the LL index axis. The error bars in the inset indicate the standard deviation of the fitting errors. (d) The energy-momentum dispersion determined by the Rashba parameters from the experimental results. The blue and red curves represent two spin-split bands with opposite spin direction. The solid lines are the Fermi levels for S1-S7 calculated by the Rashba model. 
to sample S1 and samples S3-S6 (see Appendix B). Only the oscillation frequency was obtained for S7, however. All of the fitting parameters, $F, m^{*}$, and $T_{D}$, are tabulated in Table I.

Figures 3(a) and 3(b) show $-d^{2} R_{x x} / d B^{2}$ as a function of $B$ for S5 and S7, the maxima and minima of which correspond to the oscillation maxima and minima. Besides the high-frequency oscillations from the OFS in high-field, low-frequency oscillations from the IFS can be observed in low field, as indicated by the blue arrows. Figure 3(c) shows the LL fan diagram for the IFS. Because the SdH oscillations from the IFS for S5 and S7 are very close to the regime of $\rho_{x x} \approx \rho_{x y}$, we carefully assigned the LL integer $n$, as discussed in Appendix E. For S5, the LL integer $n$ is assigned to the maxima of the $\mathrm{SdH}$ oscillations, and in the low-field part for S7, the LL integer $n$ is assigned to the maxima of the SdH oscillations, while in the high-field part, the LL integer $n$ is assigned to the minima of the $\mathrm{SdH}$ oscillations. The linear fittings yield the frequencies $2.81 \pm 0.10$ and $5.93 \pm 0.01 \mathrm{~T}$, respectively, and the intercepts with the LL index axis are at $0.756 \pm 0.048$ and $0.700 \pm 0.003$, respectively, which are very close to the region from 0.375 to 0.625 and indicate that the IFS also has a nontrivial $\pi$ Berry phase. The nontrivial $\pi$ Berry phase in the OFS and IFS is consistent with the pure bulk Rashba effect, which was first reported in BiTeI [18].

(a)

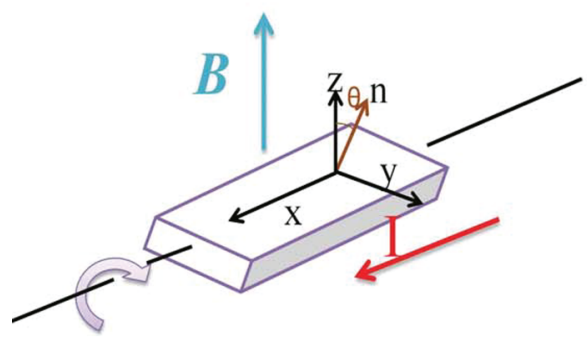

(d)

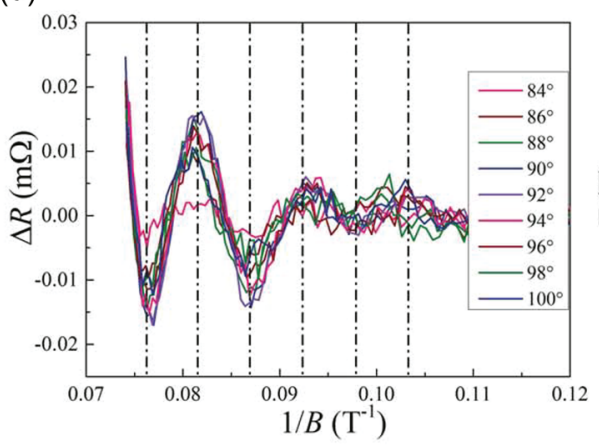

(b)

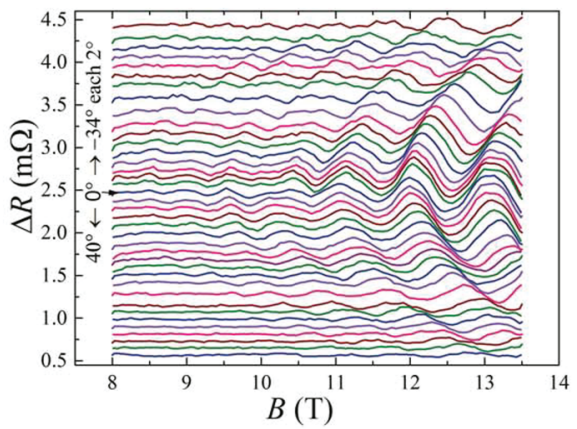

(e)

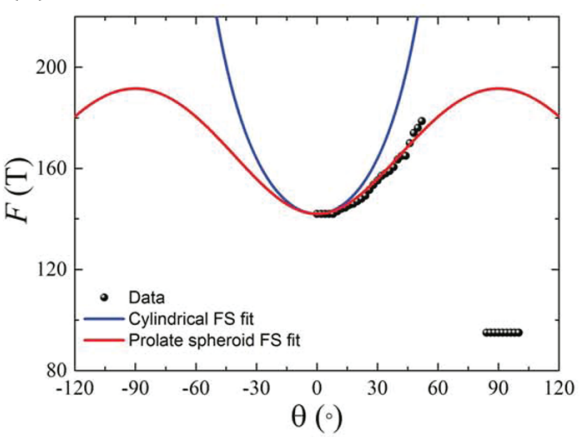

\section{Determination of Rashba parameters and Fermi levels}

We now determine the Rashba parameters and then calculate the Fermi levels of the seven samples from the Rashba model. According to the Onsager-Lifshitz equation, $F=$ ( $\hbar / 2 \pi e) A_{F}$, where $A_{F}$ is the extremal area of the cross section of the FS perpendicular to the $B$ direction. With $A_{F}=\pi k_{F}^{2}$, the Fermi wave vector is obtained, $k_{F}=\sqrt{2 e F / \hbar}$, as given in Table I. Substituting the IFS wave vector $k_{F}^{\mathrm{IFS}}$ and the OFS wave vector $k_{F}^{\mathrm{OFS}}$ of S5 and S7 into Eq. (1) yields $k_{0}=0.03158$ and $0.03006 \AA^{-1}$, respectively; the similar values indicate self-consistency of the model. Then the Rashba energy, $E_{R}=$ $\hbar^{2} k_{0}^{2} / 2 m^{*}=18.45 \mathrm{meV}$, and the Rashba constant, $\alpha_{R}=$ $2 E_{R} / k_{0}=1.20 \mathrm{eV} \AA$, are obtained with the average of the two $k_{0}$ and the average effective mass of S1-S6, which agree with the theoretical values [32,33]. Substituting the $k_{F}$ of S1-S7 into Eq. (1) yields the corresponding $E_{F}$ given in Table I. With $k_{0}$ and $m^{*}$ determined, the dispersion relation is plotted in Fig. 3(d), and the solid lines are the calculated Fermi levels for the seven samples. When the $\mathrm{SdH}$ oscillation with a single frequency exhibits a nontrivial $\pi$ Berry phase, it is easy to relate the oscillation to the topological surface state in $\mathrm{BiTeCl}$, which was recently observed by angle-resolved photoelectron spectroscopy (ARPES) [34]. The emergence of two sets of oscillations, however, rules out this possibility in this measurement. (c)

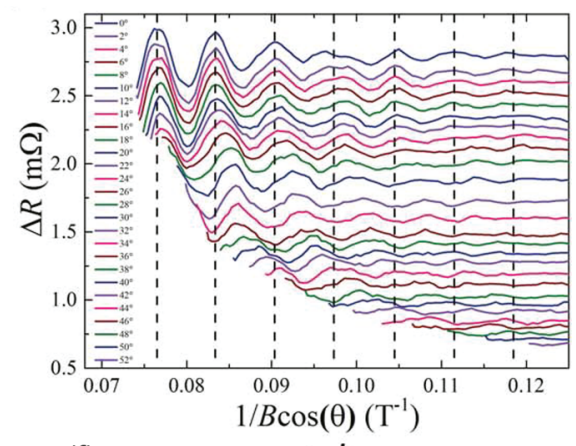

(f)

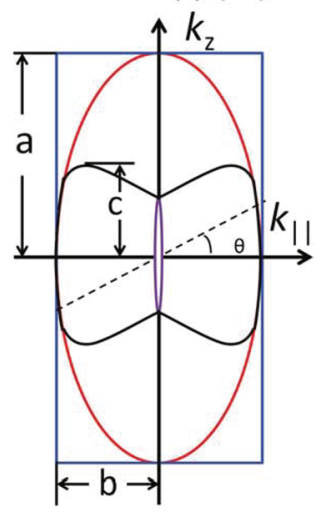

FIG. 4. (Color online) (a) Measurement configuration for angle-dependent $\mathrm{SdH}$ oscillations at $2.5 \mathrm{~K}$. The electric current and rotation axis are parallel to the $x$ direction, and $B$ is along the $\mathrm{z}$ direction. $\theta$ is defined as the angle between $B$ and the $z$ axis. The OFS SdH oscillations of S2 measured from $-34^{\circ}$ to $40^{\circ}$ (b) and from $0^{\circ}$ to $52^{\circ}$ (c) are plotted as functions of $B$ and $1 / B \cos \theta$. The oscillations are obtained after removing the polynomial background and have been vertically offset for clarity. The dashed lines indicate the oscillation period at $0^{\circ}$ on the scale with $1 / B$, which is consistent with the Landau quantization. (d) The OFS SdH oscillations of S2 around $90^{\circ}$ reveal its spherical nature. (e) Tilt angle dependence of $F$ fitted with cylindrical and prolate spheroid FS. (f) Vertical cross-sectional view of the 3D FS cut along the $k_{z}$ direction for S2. The blue rectangle denotes the cylindrical FS of a 2D electronic system, and the red ellipse denotes a prolate spheroid FS. The black and purple solids represent the OFS and IFS, which are proposed to explain the observations in Figs. 4(b)-4(e). The dashed line indicates the extremal cross section of the FS perpendicular to $B$. 
Furthermore, as tabulated in Table I, the carrier densities calculated from the $\mathrm{SdH}$ oscillations with the 3D model, $n=\left(1 / 3 \pi^{2}\right)(2 e F / \hbar)^{3 / 2}$, are consistent with the Hall effect measurements on the typical sample $\mathrm{S} 4,8.87 \times 10^{18} \mathrm{~cm}^{-3}$, which also indicates the bulk origin of the oscillation.

\section{Resolution of a 3D Fermi surface}

To resolve the 3D FS topology, the SdH oscillations were measured over an extended range of angles with the measurement configuration shown in Fig. 4(a). The oscillations exhibit symmetry in $\pm \theta$ [Fig. 4(b)], which corresponds to the symmetry of the FS. With increasing tilt angle $|\theta|$, the number of observed oscillation periods becomes less and the amplitude diminishes, which is similar to the behavior of a two-dimensional (2D) electronic system. Figure 4(c) plots the oscillations as a function of $1 / B \cos \theta$ from $0^{\circ}$ to $52^{\circ}$. From $0^{\circ}$ to $12^{\circ}$, the oscillation can be reasonably described by a $2 \mathrm{D}$ $\mathrm{FS}$, in which the period of oscillation depends on $1 / B \cos \theta$, but as the tilt angle increases further, the oscillations deviate from the expectation for a 2D FS. While the oscillation signal cannot be extracted for angles between $56^{\circ}$ and $82^{\circ}$, clear oscillations are again visible for angles around $90^{\circ}$ with a spherical $\mathrm{FS}$ character, depending on $1 / B$ rather than $1 / B \cos \theta$, however, with amplitude much smaller than those around $0^{\circ}$ [Fig. $4(\mathrm{~d})$ ].

Now we propose a quantitative 3D FS topology which captures the above feature of the oscillation. Figure 4(e) shows the angle-dependent oscillation frequency. The experimental data (black solid circles) deviate from the cylindrical FS for the 2D electronic system represented by the blue solid line $\left(F_{(\theta)} \propto \frac{1}{\cos \theta}\right)$, but the data can be fitted well by a prolate spheroid FS (red solid line) when $\theta<52^{\circ}$ $\left(F=\frac{\hbar b^{2}}{2 e} \sqrt{\left(\frac{a}{b}\right)^{2} \sin ^{2} \theta+\cos ^{2} \theta}\right.$, see Appendix C), with a major axis $a=119.6 \times 10^{-3} \AA^{-1}$ and a minor axis $b=65.69 \times$ $10^{-3} \AA^{-1}$, located on the $k_{z}$ axis and the $k_{\|}$plane of the momentum space, respectively. The spherical FS behavior around $90^{\circ}$ may be caused by the hollow shape of the OFS near zero momentum, which makes the extremal cross-sectional area constant around $90^{\circ}$. The half-height of the OFS, $c \approx$ $34.5 \times 10^{-3} \AA^{-1}$, is estimated by treating the extremal cross section of the OFS as a rectangle. The vertical cross-sectional view of the 3D FS is shown in Fig. 4(f).

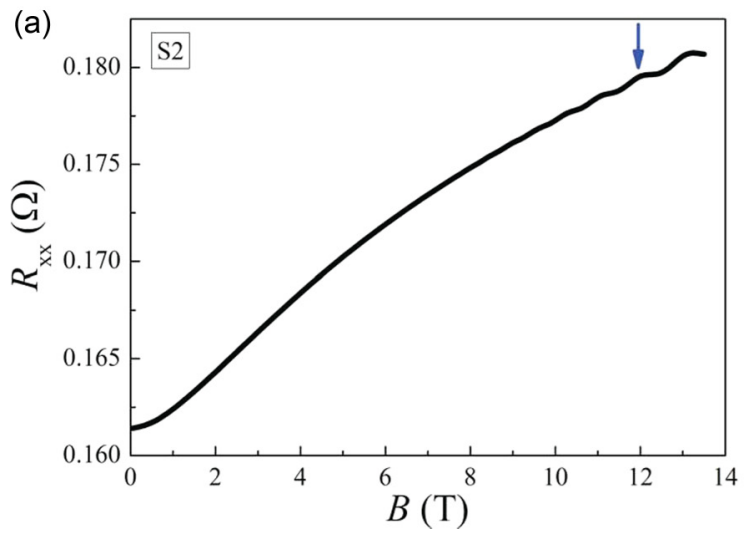

\section{CONCLUSIONS}

In this work we studied the $\mathrm{SdH}$ oscillations from the bulk Rashba spin-split conduction band of BiTeCl. The transition from two-frequency to single-frequency oscillation reveals the topological transition from a spindle-torus to a ring-torus FS. The momentum offset, Rashba energy, and Rashba coupling constant have been determined for giant Rashba spin splitting in BiTeCl. Both the inner and the outer Fermi surfaces have a nontrivial $\pi$ Berry phase. Angle-dependent oscillation measurements reveal the three-dimensional FS topology when the Fermi level lies in the vicinity of the Dirac point.

Note added. (1) Initially, we observed quantum oscillations in $\mathrm{BiTeCl}$ with a single frequency and nontrivial Berry phase, and attributed this oscillation to a topological surface state in an earlier report [35]. In this paper, by combining the Hall measurements, the Fermi surface tuning, and more extensive angledependent $\mathrm{SdH}$ measurements, we find that the oscillations originate from the bulk and that the previous observation of an $\mathrm{SdH}$ oscillation with a single frequency corresponds to the extremal case when the Fermi level is located in the vicinity of the Dirac point, where the oscillation from the IFS disappears. Recently we became aware of another two works which also conclude that $\mathrm{SdH}$ oscillations in $\mathrm{BiTeCl}$ originate from the bulk [36,37]. Our work is different from Refs. [36] and [37], however, as we observe oscillations from both the IFS and the OFS in lower magnetic field, the topological transition of the FS by tuning the carrier density with the self-doping effect, and the nontrivial $\pi$ Berry phase of both the IFS and OFS. Furthermore, we have used angle-dependent measurements to resolve the 3D Fermi surface. (2) A topological transition of the FS was also observed in BiTeI, but the relative position of the Fermi level and the band-crossing point were tuned by pressure, which modifies the band structure [38]. Very recently we have become aware of similar work by Ye et al. [39].

\section{ACKNOWLEDGMENTS}

This work is supported by the Australian Research Council (ARC) through an ARC Discovery Project (DP130102956, X.L.W.) and an ARC Professorial Future Fellowship project (FT130100778, X.L.W.). M.S.F. is supported in part by an ARC Laureate Fellowship.

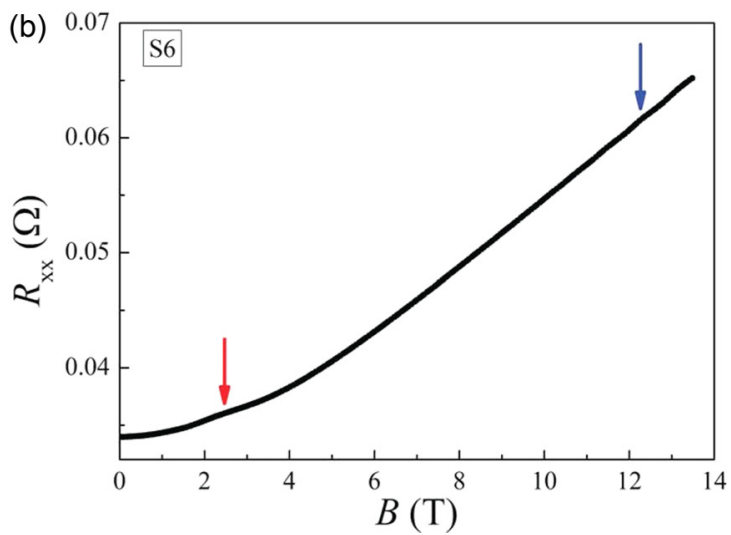

FIG. 5. (Color online) (a, b) Typical SdH oscillations without background subtraction. 

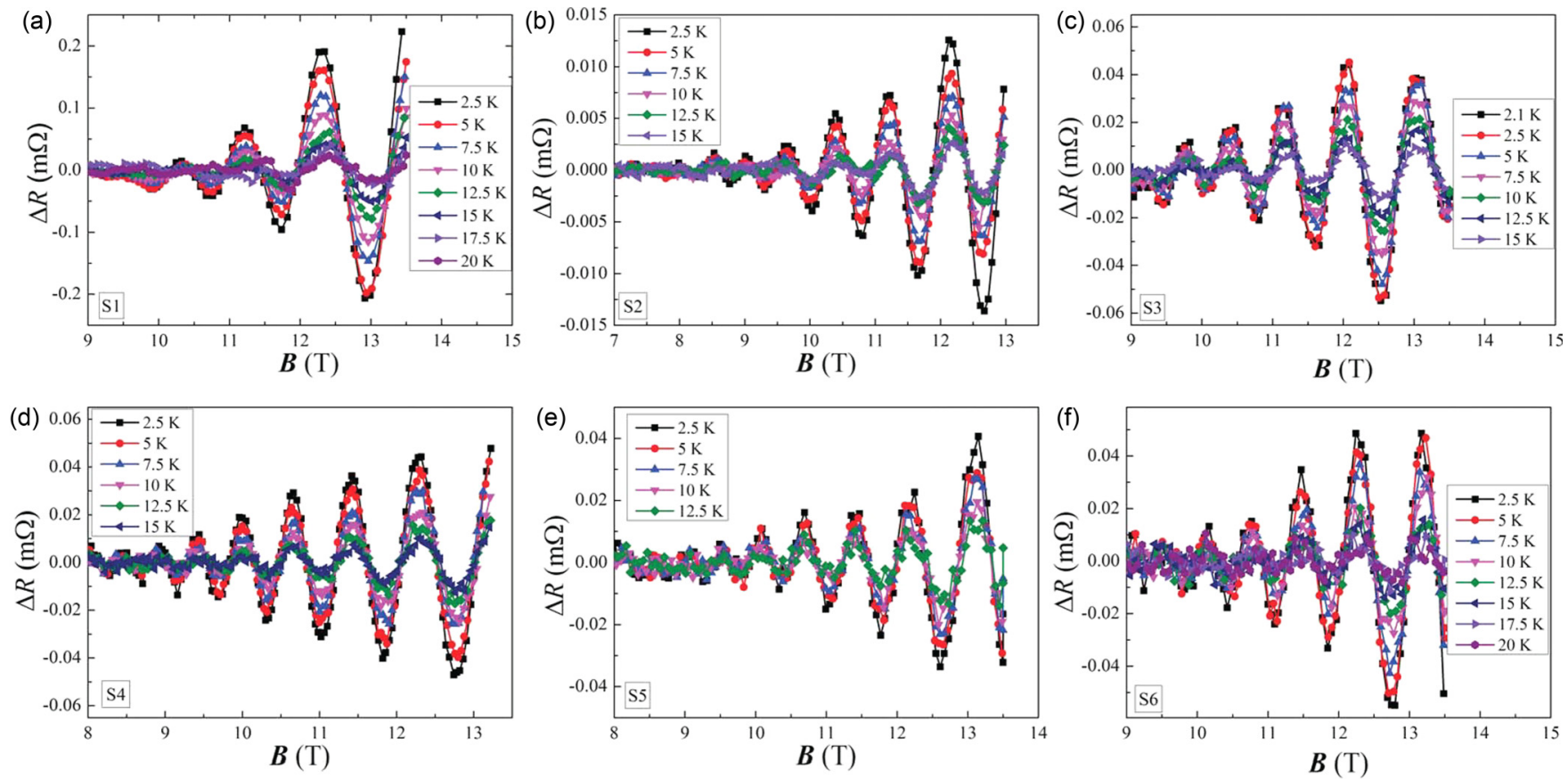

FIG. 6. (Color online) (a)-(f) SdH oscillations measured at various temperatures for S1-S6. The oscillations are obtained after removing the background.

\section{APPENDIX A: TYPICAL SdH OSCILLATIONS WITHOUT BACKGROUND SUBTRACTION}

Figures 5(a) and 5(b) show two typical Shubnikov-de Haas oscillations without background subtraction observed in measurements. In Fig. 5(a) clear SdH oscillations can only be observed in high magnetic field, as indicated by the blue arrow, while in Fig. 5(b), weak SdH oscillations can be observed in both low and high magnetic field, as indicated by the blue and red arrows, respectively.
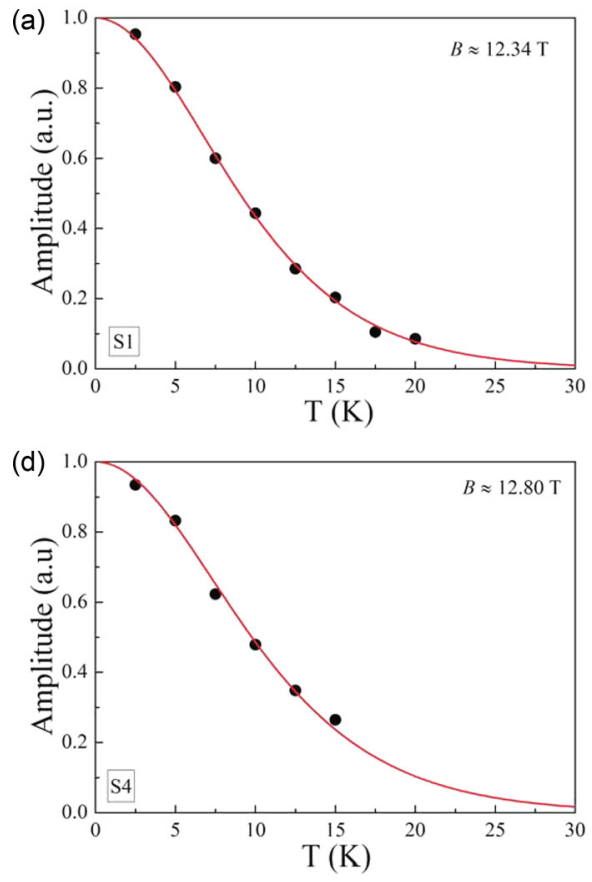

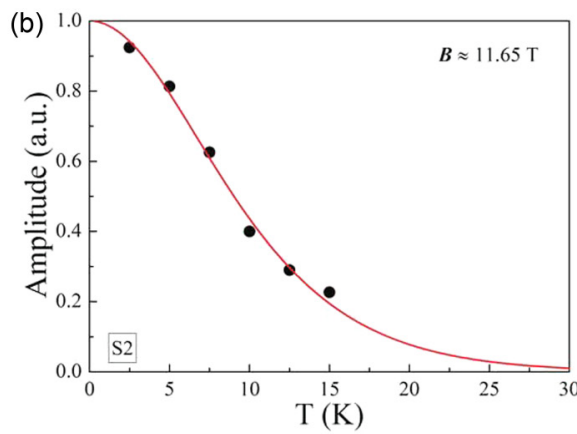

\section{APPENDIX B: STANDARD SdH OSCILLATION ANALYSIS WITH LK FOMULA OF QUANTUM OSCILLATIONS OF MORE SAMPLES}

In addition to the standard $\mathrm{SdH}$ oscillation analysis with the LK formula for quantum oscillations from the OFS of S2 in Figs. 2(a), 2(b), and 2(d), the same analysis method used in the main text was used for the other samples, except for S7, as shown in Figs. 6-8. In combination with the LL fan diagram, the effective mass $m^{*}$, oscillation frequency $F$, and
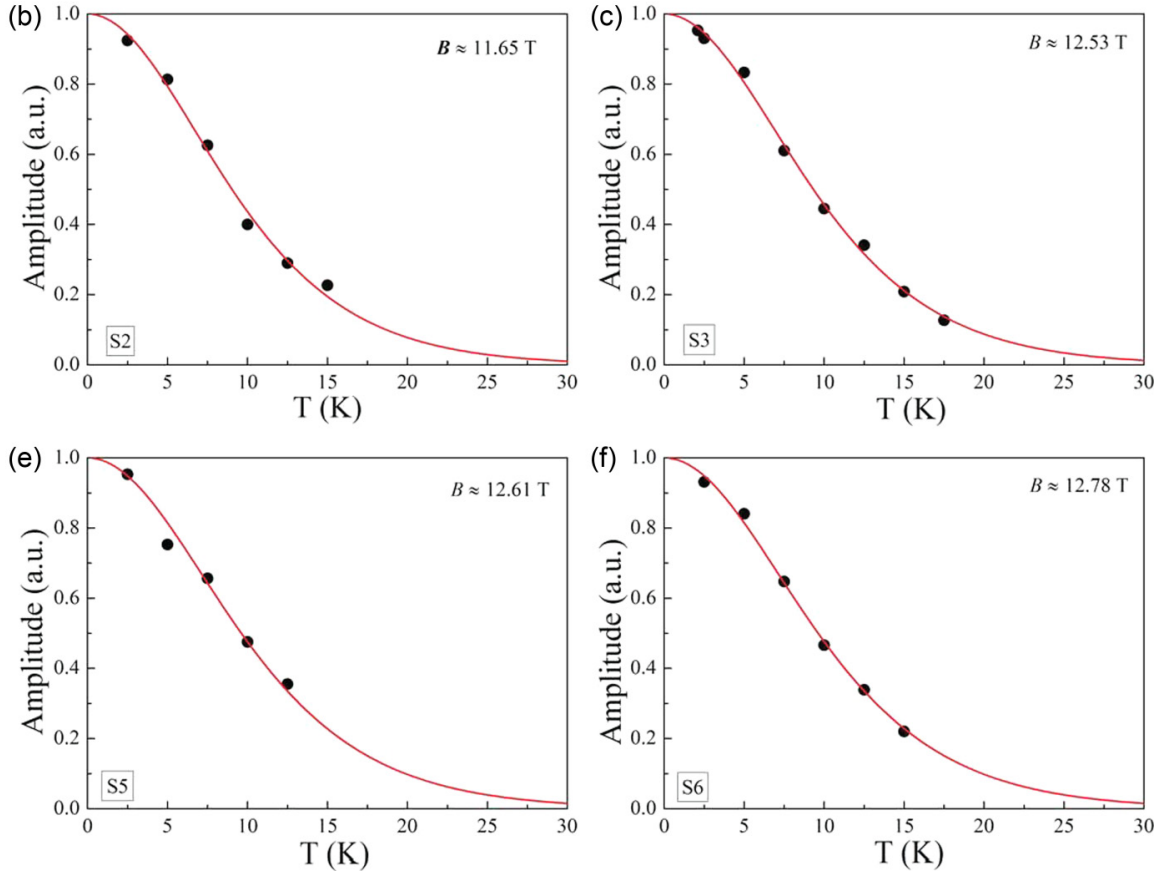

FIG. 7. (Color online) (a)-(f) Temperature dependence of the oscillation amplitude for S1-S6. The red solid lines are the fitting with the thermal damping factor, $\frac{2 \pi^{2} k_{B} T m^{*} / \hbar e B}{\sinh \left(2 \pi^{2} k_{B} T m^{*} / \hbar e B\right)}$, which yields the effective mass. 

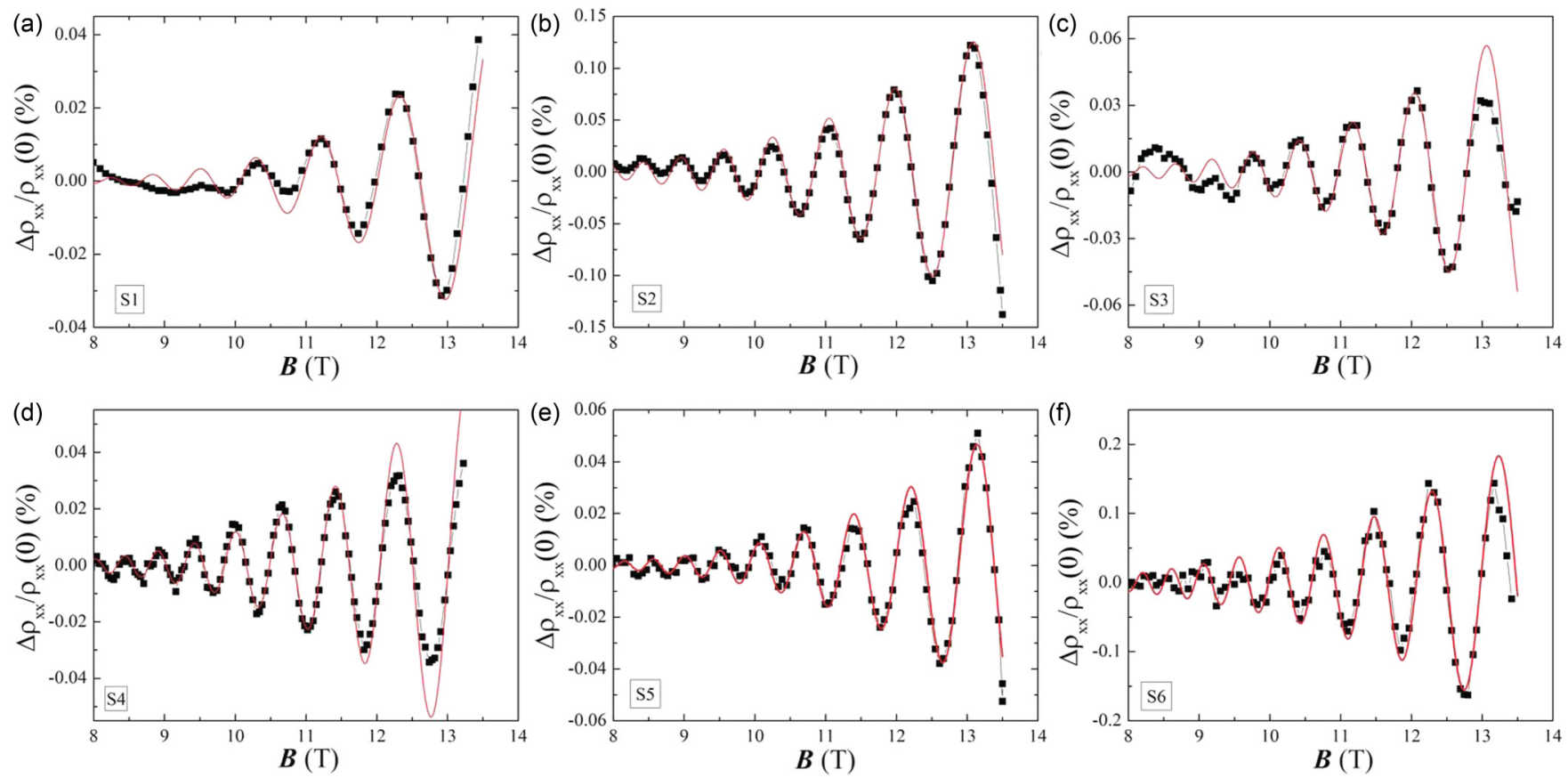

FIG. 8. (Color online) (a)-(f) LK fitting of SdH oscillations at 2.5 K for S1-S6, which yields the Dingle temperature.

Dingle temperature $T_{D}$ can be extracted, as shown in Table I in the main text.

\section{APPENDIX C: ANGLE-DEPENDENT SdH OSCILLATION FREQUENCY OF A PROLATE SPHEROID FS}

According to the angle-dependent $\mathrm{SdH}$ oscillation in Fig. 4(e), the FS deviates from the cylindrical shape for a two-dimensional electronic system. To describe the 3D FS in $\mathrm{BiTeCl}$, several FS geometries were tried, and it was found that a prolate-spheroid-based FS can fit the angle-dependent oscillation frequency reasonably well when $\theta \leqslant 52^{\circ}$ (see Fig. 9). The equation of the prolate spheroid, with two semiaxes with length $b$ in the $k_{\|}$plane and one semiaxis $a$ in the $k_{z}$ direction, can be expressed as

$$
\frac{x^{2}+y^{2}}{b^{2}}+\frac{z^{2}}{a^{2}}=1,(a>b) .
$$

If the extremal cross section of the FS perpendicular to the magnetic field is an ellipse $(\theta>0)$ or a circle $(\theta=0)$, its equation can be expressed as

$$
\frac{x^{2}}{b^{2}}+\frac{y^{2}}{a^{2} \sin ^{2} \theta+b^{2} \cos ^{2} \theta}=1(\theta \text { is the tilt angle }) .
$$

Therefore, the extremal cross-sectional area of the FS perpendicular to the magnetic field is

$$
A_{F}=\pi b^{2} \sqrt{\left(\frac{a}{b}\right)^{2} \sin ^{2} \theta+\cos ^{2} \theta} .
$$

Because $F=(\hbar / 2 \pi e) A_{\mathrm{F}}, F$ as a function of $\theta$ can be expressed as

$$
F=\frac{\hbar b^{2}}{2 e} \sqrt{\left(\frac{a}{b}\right)^{2} \sin ^{2} \theta+\cos ^{2} \theta} .
$$

\section{APPENDIX D: ANGULAR DEPENDENCE OF SdH OSCILLATIONS FROM OFS OF S4}

Besides the angular dependence of the SdH oscillation analysis in sample $\mathrm{S} 2$, similar measurements were also carried out on sample S4. Figure 10(a) shows the quantum oscillations from $-6^{\circ}$ to $46^{\circ}$, in which the number of the observed oscillation periods becomes less as the tilt angle increases and

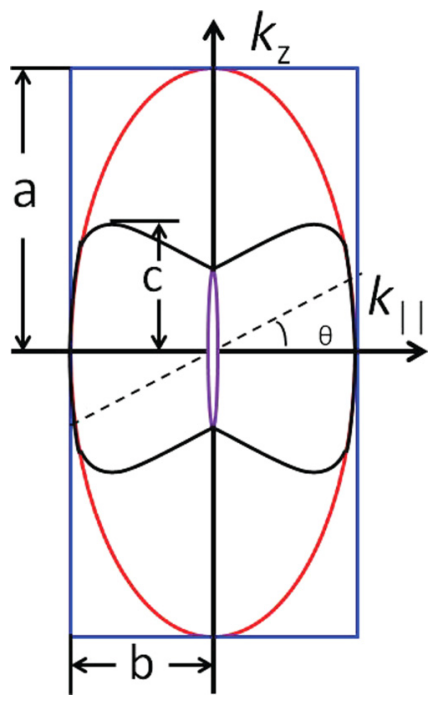

FIG. 9. (Color online) Vertical cross-sectional view of the 3D FS cut along the $k_{z}$ direction for $\mathrm{S} 2$. The blue rectangle denotes the cylindrical FS of a 2D electronic system, and the red ellipse denotes a prolate spheroid FS with major axis $a=119.6 \times 10^{-3} \AA^{-1}$ and minor axis $b=65.69 \times 10^{-3} \AA^{-1}$, respectively. The black and purple solids represent the OFS and IFS, which is proposed to explain the observations in Figs. 4(b)-4(e). The dashed line indicates the extremal cross section of the FS perpendicular to the magnetic field. 

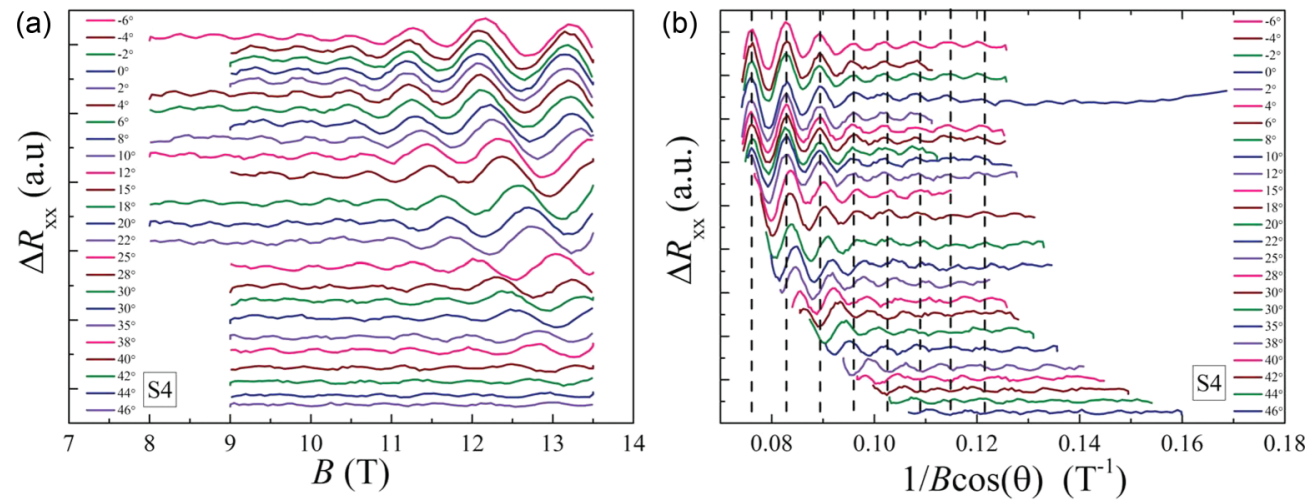

FIG. 10. (Color online) (a) SdH oscillations from the OFS of S4 at various angles after removing the background. The curves are vertically offset for clarity. (b) $\mathrm{SdH}$ oscillations in (a) are plotted on the $1 / B$ scale. The dashed lines indicate the oscillation period.
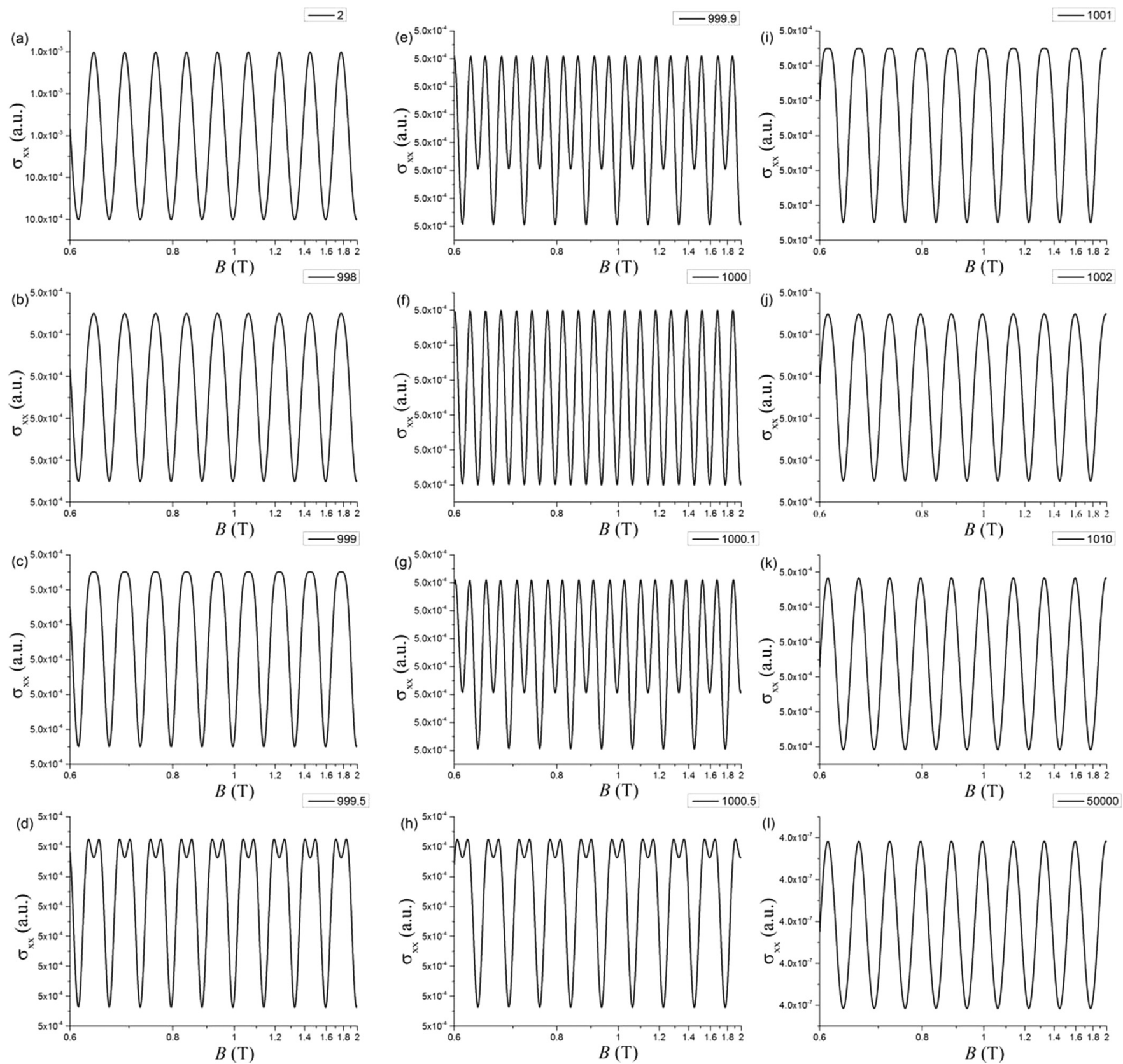

FIG. 11. (a)-(1) Longitudinal conductivity $\sigma_{x x}$ as a function of magnetic field on the reciprocal scale is plotted for various $\rho_{x y} / \rho_{x x}$ ratios. Among them, $\rho_{x y} \approx 5000 \rho_{x x}$, and $2 \rho_{x x}$ corresponds to $\rho_{x x} \ll \rho_{x y}$ and $\rho_{x x} \gg \rho_{x y}$, respectively. 

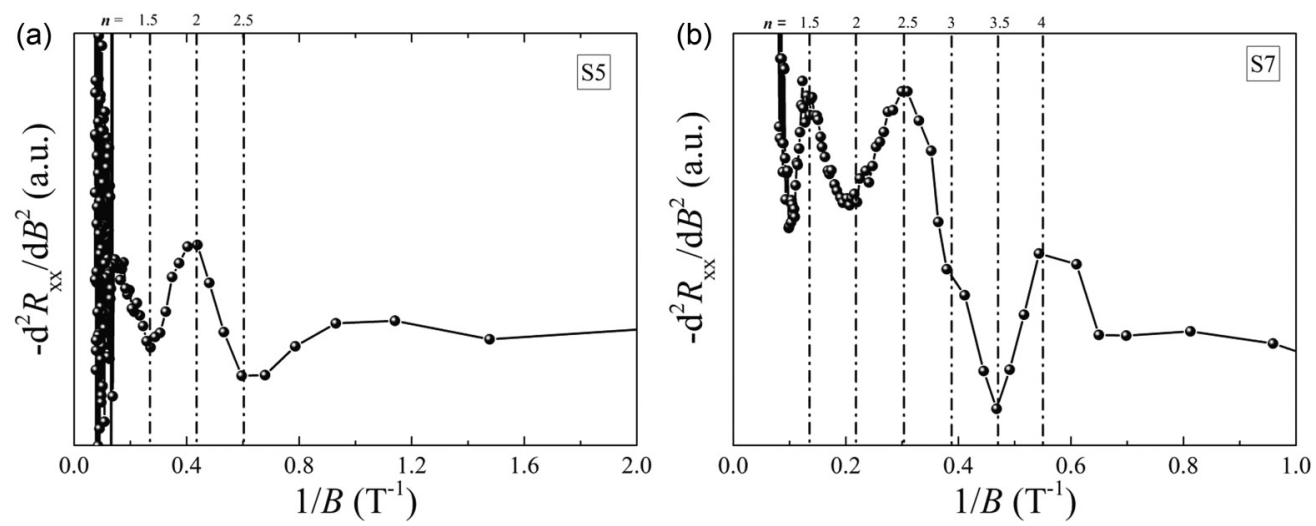

FIG. 12. $-d^{2} R_{x x} / d B^{2}$ as a function of $1 / B$ for samples S5 and S7. The dashed-dotted lines mark the minima and maxima of the SdH oscillations, indicating the oscillatory periods on the $1 / B$ scale.

the oscillation amplitude diminishes, in a similar way to $\mathrm{S} 2$. Figure 10(b) shows the same quantum oscillations as Fig. 10(a) on the $1 / B$ scale. The dashed lines in Fig. 10(b) indicate the period of oscillations at $0^{\circ}$. It can be seen more clearly that the oscillations from the OFS of S4 exhibit a stronger two-dimensional feature compared with those of S2, which agrees with the topological transition of the FS. Because the Fermi level of S4 is higher than that of S2, the Fermi surface is more like the spindle-torus shape and the OFS is more cylindrical in shape.

\section{APPENDIX E: ASSIGNMENT OF LANDAU-LEVEL INDEX AND DETERMINATION OF BERRY PHASE}

The measurements of both longitudinal resistivity and Hall resistivity are important not only for calculating the carrier density and Hall mobility, but also for assigning the Landaulevel (LL) index and determining the Berry phase. Because of $\sigma_{x x}=\frac{\rho_{x x}}{\rho_{x x}^{2}+\rho_{x y}^{2}}$, while for $\rho_{x x} \gg \rho_{x y}$, the minima and maxima of the oscillations in the longitudinal conductivity $\sigma_{x x}$ are out of phase with those in the longitudinal $\rho_{x x}$, and the LL integer $n$ is assigned to the oscillatory maxima of $\rho_{x x}$; for the case of $\rho_{x x} \ll \rho_{x y}$, the minima and maxima of the oscillations in $\sigma_{x x}$ are in phase with those in $\rho_{x x}$, and the LL integer $n$ is assigned to oscillatory minima of $\rho_{x x}$. How the phase factor shifts when $\rho_{x x} \approx \rho_{x y} \mid$ is still unclear, however. To answer this question, we establish a simple model to estimate the phase shift window. Assumption 1: $\rho_{x x}=\Delta \rho_{x x}+\rho_{x x}^{\text {const }}, \Delta \rho_{x x} \ll \rho_{x x}^{\text {const }}$, where

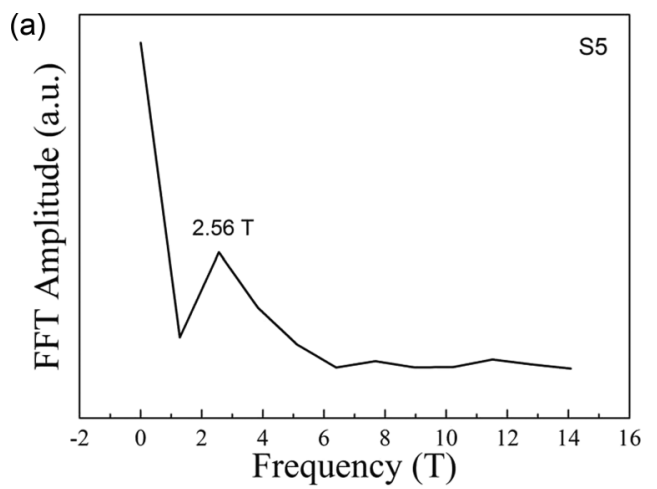

$\Delta \rho_{x x}$ is the oscillatory part of $\rho_{x x}$, and $\rho_{x x}^{\text {const }}$ is the background and assumed to be a constant for simplicity. (In our case as shown in Fig. 8, $\frac{\Delta \rho_{x x}}{\rho_{x x}^{\text {const }}} \approx \frac{\Delta \rho_{x x}}{\rho_{x x}}<\frac{1}{1000}$ ).

Assumption 2: The oscillations in the experiment come from $\Delta \rho_{x x}$.

Now when $\rho_{x x} \ll \rho_{x y}$, which is always the case in a oneband model when $\mathrm{SdH}$ oscillation is observed $\left(\rho_{x y}=\rho_{x x} *\right.$ $\mu B$, with $\mu B \gg 1$ in order to have $\mathrm{SdH}$ oscillations), then we can write

$$
\sigma_{x x}=\frac{\rho_{x x}}{\rho_{x x}^{2}+\rho_{x y}^{2}} \approx \frac{\rho_{x x}^{\text {const }}}{\rho_{x y}^{2}}+\frac{\Delta \rho_{x x}}{\rho_{x y}^{2}} .
$$

So $\sigma_{x x}$ is in phase with $\rho_{x x}$.

When $\rho_{x x} \gg \rho_{x y}$ (which is possible in a multiband case where there is a dominant low-mobility band present), then we can write

$$
\sigma_{x x}=\frac{\rho_{x x}}{\rho_{x x}^{2}+\rho_{x y}^{2}} \approx \frac{1}{\rho_{x x}}
$$

so $\sigma_{x x}$ is out of phase with $\rho_{x x}$.

In the case of $\rho_{x x} \approx \rho_{x y}$, because of $\frac{\Delta \rho_{x x}}{\rho_{x x}^{\text {const }}}<\frac{1}{1000}$ as shown in Fig. 8, $\rho_{x x}$ can be written approximately as $\rho_{x x}=$ $1000+\cos (50 / B)$, where the oscillation amplitude is assume to be 1 for simplicity, and $2 \pi F$ and the constant part is set to 50 and 1000 , respectively. As the $\rho_{x y} / \rho_{x x}$ ratio is varied from 2 to $50,000, \sigma_{x x}$ is plotted as a function of magnetic field on the reciprocal scale, as shown in the Fig. 11. For $\rho_{x x} \ll \rho_{x y}$, i.e.,

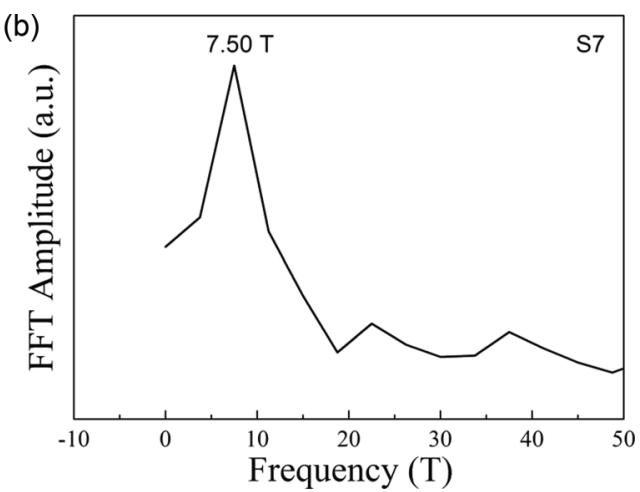

FIG. 13. Fast Fourier transform spectra of SdH oscillation frequency for samples S5 (a) and S7 (b). 
$\rho_{x y} \approx 5000 \rho_{x x}$ [(1) of Fig. 11], and $\rho_{x x} \gg \rho_{x y}$, i.e., $\rho_{x y} \approx$ $2 \rho_{x x}$ [(a) of Fig. 11], the phase factors in $\sigma_{x x}$ differ by $180^{\circ}$. When $\rho_{x x} \approx \rho_{x y}$, it was found the phase changes only in a very narrow window. The change in $\rho_{x y}$ is on the order of $2 \Delta \rho_{x x}$, which equals $2 \cos (50 / B) \approx 2$, and in this case, will shift the phase by $180^{\circ}$. When $999 \rho_{x x} \leqslant \rho_{x y} \leqslant 1001 \rho_{x x}$, the period of oscillation becomes halved.

As in the experimental data shown in Figs. 1(b) and 1(c), the regime of $\rho_{x x} \approx \rho_{x y}$ is around 3-5 T. For the SdH oscillations from the OFS of S1 to S7, $\rho_{x x}<\rho_{x y}$, so the LL integer $n$ is assigned to the minima of $\mathrm{SdH}$ oscillations. The $\mathrm{SdH}$ oscillations from the IFS of S5 can be categorized into the regime of $\rho_{x x}>\rho_{x y}$, so the LL integer $n$ is assigned to the maxima of the SdH oscillations, as shown in Fig. 12(a). For the $\mathrm{SdH}$ oscillations from the IFS of S7, because the oscillations extend from the low field, around $1.5 \mathrm{~T}$, to high field, around
$8 \mathrm{~T}$, in the low-field part the LL integer $n$ is assigned to the minima of the $\mathrm{SdH}$ oscillations, and in the high-field part, the LL integer $n$ is assigned to the maxima of the SdH oscillations, as shown in Fig. 12(b). The dashed-dotted lines indicate the minima and maxima of oscillations and also indicate that the oscillatory periods are on the $1 / B$ scale, which agrees with the Landau quantization.

\section{APPENDIX F: EXTRACTION OF SdH OSCILLATION FREQUENCY WITH FAST FOURIER TRANSFORM}

Figure 13 shows the $\mathrm{SdH}$ oscillation frequencies for S5 and S7 yielded by the respective fast Fourier transforms (FFTs). They are quite close to the oscillation frequencies extracted from Landau-level fan diagram analysis, 2.81 and $5.93 \mathrm{~T}$ for S5 and S7, respectively.
[1] D. J. Griffiths, Introduction to Electrodynamics, 3rd ed. (Prentice Hall, Upper Saddle River, NJ, 1998).

[2] V. Galitski and I. B. Spielman, Spin-orbit coupling in quantum gases, Nature (London) 494, 49 (2013).

[3] G. Dresselhaus, Spin-orbit coupling effects in zinc blende structures, Phys. Rev. 100, 580 (1955).

[4] E. I. Rashba, Properties of semiconductors with an extremum loop. 1. Cyclotron and combinational resonance in a magnetic field perpendicular to the plane of the loop, Sov. Phys. Solid State 2, 1109 (1960).

[5] Y. A. Bychkov and E. I. Rashba, Porperties of a 2D electron gas with lifted spectral degeneracy, JETP Lett. 39, 78 (1984).

[6] K. Ishizaka, M. S. Bahramy, H. Murakawa, M. Sakano, T. Shimojima, T. Sonobe, K. Koizumi, S. Shin, H. Miyahara, A. Kimura, K. Miyamoto, T. Okuda, H. Namatame, M. Taniguchi, R. Arita, N. Nagaosa, K. Kobayashi, Y. Murakami, R. Kumai, Y. Kaneko, Y. Onose, and Y. Tokura, Giant Rashba-type spin splitting in bulk BiTeI, Nat. Mater. 10, 521 (2011).

[7] J. S. Lee, G. A. H. Schober, M. S. Bahramy, H. Murakawa, Y. Onose, R. Arita, N. Nagaosa, and Y. Tokura, Optical response of relativistic electrons in the polar BiTeI semiconductor, Phys. Rev. Lett. 107, 117401 (2011).

[8] A. Crepaldi, L. Moreschini, G. Autès, C. Tournier-Colletta, S. Moser, N. Virk, H. Berger, P. Bugnon, Y. J. Chang, K. Kern, A. Bostwick, E. Rotenberg, O. V. Yazyev, and M. Grioni, Giant ambipolar rashba effect in the semiconductor BiTeI, Phys. Rev. Lett. 109, 096803 (2012).

[9] G. Landolt, S. V. Eremeev, Y. M. Koroteev, B. Slomski, S. Muff, T. Neupert, M. Kobayashi, V. N. Strocov, T. Schmitt, Z. S. Aliev, M. B. Babanly, I. R. Amiraslanov, E. V. Chulkov, J. Osterwalder, and J. H. Dil, Disentanglement of surface and bulk rashba spin splittings in noncentrosymmetric BiTeI, Phys. Rev. Lett. 109, 116403 (2012).

[10] S. V. Eremeev, I. A. Nechaev, Y. M. Koroteev, P. M. Echenique, and E. V. Chulkov, Ideal two-dimensional electron systems with a giant Rashba-type spin splitting in real materials: Surfaces of bismuth tellurohalides, Phys. Rev. Lett. 108, 246802 (2012).

[11] G. Landolt, S. V. Eremeev, O. E. Tereshchenko, S. Muff, B. Slomski, K. A. Kokh, M. Kobayashi, T. Schmitt, V. N. Strocov, J. Osterwalder, E. V. Chulkov, and J. H. Dil, Bulk and surface
Rashba splitting in single termination BiTeCl, New J. Phys. 15 085022 (2013).

[12] S. A. Wolf, D. D. Awschalom, R. A. Buhrman, J. M. Daughton, S. von Molnár, M. L. Roukes, A. Y. Chtchelkanova, and D. M. Treger, Spintronics: A spin-based electronics vision for the future, Science 294, 1488 (2001).

[13] D. Awschalom and N. Samarth, Spintronics without magnetism, Physics 2, 50 (2009).

[14] J. Sinova, D. Culcer, Q. Niu, N. A. Sinitsyn, T. Jungwirth, and A. H. MacDonald, Universal intrinsic spin hall effect, Phys. Rev. Lett. 92, 126603 (2004).

[15] L. P. Gor'kov and E. I. Rashba, Superconducting 2D system with lifted spin degeneracy: Mixed singlet-triplet state, Phys. Rev. Lett. 87, 037004 (2001).

[16] E. Bauer, G. Hilscher, H. Michor, C. Paul, E. W. Scheidt, A. Gribanov, Y. Seropegin, H. Noël, M. Sigrist, and P. Rogl, Heavy fermion superconductivity and magnetic order in noncentrosymmetric CePt3Si, Phys. Rev. Lett. 92, 027003 (2004).

[17] J. D. Sau, R. M. Lutchyn, S. Tewari, and S. Das Sarma, Generic new platform for topological quantum computation using semiconductor heterostructures, Phys. Rev. Lett. 104, 040502 (2010).

[18] V. Mourik, K. Zuo, S. M. Frolov, S. R. Plissard, E. P. A. M. Bakkers, and L. P. Kouwenhoven, Signatures of majorana fermions in hybrid superconductor-semiconductor nanowire devices, Science 336, 1003 (2012).

[19] E. Cappelluti, C. Grimaldi, and F. Marsiglio, Topological change of the fermi surface in low-density rashba gases: Application to superconductivity, Phys. Rev. Lett. 98, 167002 (2007).

[20] K. Tsutsui and S. Murakami, Spin-torque efficiency enhanced by Rashba spin splitting in three dimensions, Phys. Rev. B 86, 115201 (2012).

[21] F. Meier, V. Petrov, S. Guerrero, C. Mudry, L. Patthey, J. Osterwalder, and J. H. Dil, Unconventional Fermi surface spin textures in the $\mathrm{Bi}_{x} \mathrm{~Pb}_{1-x} / \mathrm{Ag}(111)$ surface alloy, Phys. Rev. B 79, 241408 (2009).

[22] A. R. Mellnik, J. S. Lee, A. Richardella, J. L. Grab, P. J. Mintun, M. H. Fischer, A. Vaezi, A. Manchon, E. A. Kim, N. Samarth, and D. C. Ralph, Spin-transfer torque generated by a topological insulator, Nature (London) 511, 449 (2014). 
[23] J.-V. Kim, Solid-state physics: Siphoning spins, Nature (London) 511, 418 (2014).

[24] X. Wang, Y. Du, S. Dou, and C. Zhang, Room temperature giant and linear magnetoresistance in topological insulator $\mathrm{Bi}_{2} \mathrm{Te}_{3}$ nanosheets, Phys. Rev. Lett. 108, 266806 (2012).

[25] M. Veldhorst, M. Snelder, M. Hoek, T. Gang, V. K. Guduru, X. L. Wang, U. Zeitler, W. G. van der Wiel, A. A. Golubov, H. Hilgenkamp, and A. Brinkman, Josephson supercurrent through a topological insulator surface state, Nat. Mater. 11, 417 (2012).

[26] D. Shoenberg, Magnetic Oscillations in Metals (Cambridge University Press, Cambridge, UK, 1984).

[27] G. Landwehr and E. I. Rashba, Landau Level Spectroscopy, Modern Problems in Condensed Matter Sciences Vol. 27.2 (North-Holland, Amsterdam, 1991).

[28] Y. Zhang, Y.-W. Tan, H. L. Stormer, and P. Kim, Experimental observation of the quantum Hall effect and Berry's phase in graphene, Nature (London) 438, 201 (2005).

[29] H. Murakawa, M. S. Bahramy, M. Tokunaga, Y. Kohama, C. Bell, Y. Kaneko, N. Nagaosa, H. Y. Hwang, and Y. Tokura, Detection of Berry's phase in a bulk Rashba semiconductor, Science 342, 1490 (2013).

[30] M. Veldhorst, M. Snelder, M. Hoek, C. G. Molenaar, D. P. Leusink, A. A. Golubov, H. Hilgenkamp, and A. Brinkman, Magnetotransport and induced superconductivity in Bi based three-dimensional topological insulators, Physica Status Solidi RRL 7, 26 (2013).

[31] F.-X. Xiang, X.-L. Wang, and S.-X. Dou, Transport evidence for the coexistence of the topological surface state and a two-dimensional electron gas in BiSbTe3 topological insulator, arXiv: 1404.7572 .
[32] Z. Zhu, Y. Cheng, and U. Schwingenschlögl, Orbital-dependent Rashba coupling in bulk BiTeCl and BiTeI, New J. Phys. 15, 023010 (2013).

[33] I. P. Rusinov, I. A. Nechaev, S. V. Eremeev, C. Friedrich, S. Blügel, and E. V. Chulkov, Many-body effects on the Rashbatype spin splitting in bulk bismuth tellurohalides, Phys. Rev. B 87, 205103 (2013).

[34] Y. L. Chen, M. Kanou, Z. K. Liu, H. J. Zhang, J. A. Sobota, D. Leuenberger, S. K. Mo, B. Zhou, S. L. Yang, P. S. Kirchmann, D. H. Lu, R. G. Moore, Z. Hussain, Z. X. Shen, X. L. Qi, and T. Sasagawa, Discovery of a single topological Dirac fermion in the strong inversion asymmetric compound $\mathrm{BiTeCl}$, Nat. Phys. 9, 704 (2013).

[35] F. X. Xiang, X. L. Wang, and S. X. Dou, Transport evidence of robust topological surface state in $\mathrm{BiTeCl}$ single crystals, the first strong inversion asymmetric topological insulator, arXiv:1401.6732.

[36] C. Martin, A. V. Suslov, S. Buvaev, A. F. Hebard, P. Bugnon, H. Berger, A. Magrez, and D. B. Tanner, Unusual Shubnikov-de Haas oscillations in BiTeCl, Phys. Rev. B 90, 201204 (2014).

[37] F. Chen, D. Zhao, Z. J. Xiang, C. Shang, X. G. Luo, B. Y. Pan, S. Y. Li, T. Wu, and X. H. Chen, Quantum oscillations in Rashba semiconductor BiTeCl, Phys. Rev. B 90, 201202 (2014).

[38] D. VanGennep, S. Maiti, D. Graf, S. W. Tozer, C. Martin, H. Berger, D. L. Maslov, and J. J. Hamlin, Pressure tuning the Fermi level through the Dirac point of giant Rashba semiconductor BiTeI, J. Phys.: Condens. Matter 26, 342202 (2014).

[39] L. Ye, J. G. Checkelsky, F. Kagawa, and Y. Tokura, Transport signatures of Fermi surface topology change in BiTeI, Phys. Rev. B 91, 201104 (2015). 